\title{
Hydrodynamic characteristics of a pilot-scale dual fluidized bed with continuous feeding and discharging of solids: Experiment and 3D simulation
}

Zheng Zou, † Zhan Du, † Goqiang Shao, $†$ Qi Liu,,+ Zhaohui Xie, $†$ Hongzhong $L i, *,+\ldots$ Qingshan Zhu,,$+\neq$

$\uparrow$ State Key Laboratory of Multi-phase Complex Systems, Institute of Process Engineering, Chinese Academy of Sciences, P.O. Box 353, Beijing 100190, PR China

† University of Chinese Academy of Sciences, Beijing 100049, PR China

*hzli@ipe.ac.cn 
Supplement S1. The laboratory photograph of pilot-scale DFB plant.

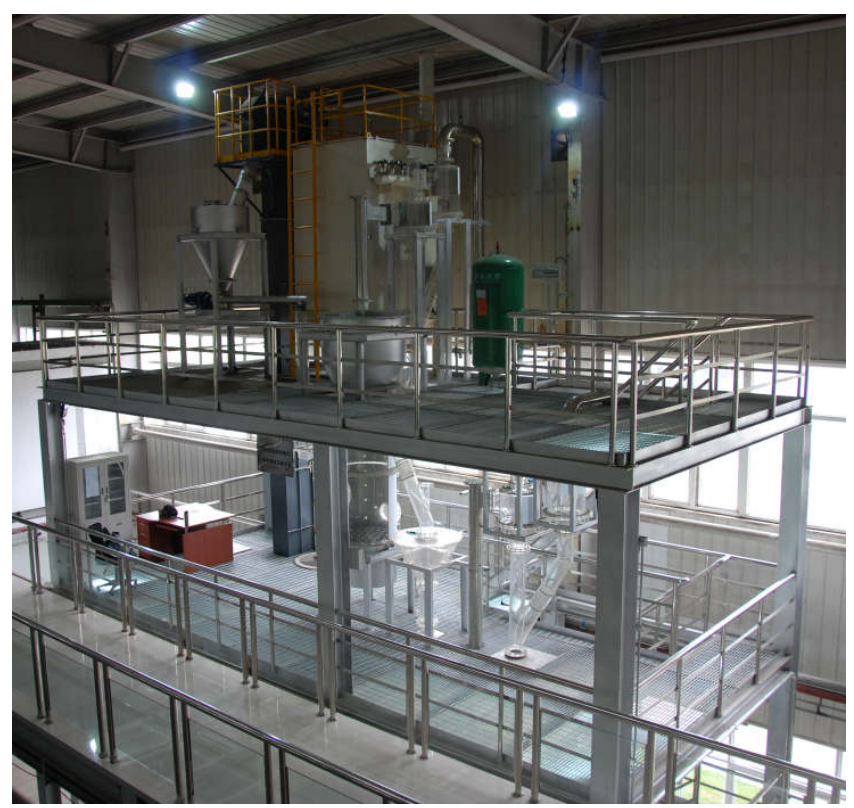

Figure S1. The laboratory photograph of pilot-scale DFB plant.

Supplement S2. The simplified kinetic theory of granular flow (KTGF) governing equations used for the CFD model.

Table S2. Governing equations for two-fluid model.

Continuity equation $(\mathrm{k}=\mathrm{g}, \mathrm{s})$

$\frac{\partial}{\partial t}\left(\varepsilon_{\mathrm{k}} \rho_{\mathrm{k}}\right)+\nabla \cdot\left(\varepsilon_{\mathrm{k}} \rho_{\mathrm{k}} \vec{u}_{\mathrm{k}}\right)=0$

Momentum equation

$$
\begin{aligned}
& \frac{\partial}{\partial t}\left(\varepsilon_{\mathrm{g}} \rho_{\mathrm{g}} \vec{u}_{\mathrm{g}}\right)+\nabla \cdot\left(\varepsilon_{\mathrm{g}} \rho_{\mathrm{g}} \vec{u}_{\mathrm{g}} \vec{u}_{\mathrm{g}}\right) \\
& \quad=-\varepsilon_{\mathrm{g}} \nabla p+\varepsilon_{\mathrm{g}} \rho_{\mathrm{g}} \vec{g}+\nabla \cdot \overline{\bar{\tau}}_{\mathrm{g}}+\beta\left(\vec{u}_{\mathrm{s}}-\vec{u}_{\mathrm{g}}\right) \\
& \frac{\partial}{\partial t}\left(\varepsilon_{\mathrm{s}} \rho_{\mathrm{s}} \vec{u}_{\mathrm{s}}\right)+\nabla \cdot\left(\varepsilon_{\mathrm{s}} \rho_{\mathrm{s}} \vec{u}_{\mathrm{s}} \vec{u}_{\mathrm{s}}\right) \\
& \quad=-\varepsilon_{\mathrm{s}} \nabla p-\nabla p_{\mathrm{s}}+\varepsilon_{\mathrm{s}} \rho_{\mathrm{s}} \vec{g}+\nabla \cdot \overrightarrow{\bar{\tau}}_{\mathrm{s}}+\beta\left(\vec{u}_{\mathrm{g}}-\vec{u}_{\mathrm{s}}\right)
\end{aligned}
$$


Stress-strain tensor for gas and solid phases $(\mathrm{k}=\mathrm{g}, \mathrm{s})$

$$
\overline{\bar{\tau}}=\varepsilon_{\mathrm{k}} \mu_{\mathrm{k}}\left(\nabla \vec{u}_{\mathrm{k}}+\nabla \vec{u}_{\mathrm{k}}^{\mathrm{T}}\right)-\frac{2}{3} \varepsilon_{\mathrm{k}} \mu_{\mathrm{k}}\left(\nabla \cdot \vec{u}_{\mathrm{k}}\right) \overline{\bar{I}}
$$

Solid pressure

$$
p_{\mathrm{s}}=\varepsilon_{\mathrm{s}} \rho_{\mathrm{s}} \Theta_{\mathrm{s}}+2 \varepsilon_{\mathrm{s}}^{2}(1+e) \rho_{\mathrm{s}} g_{0} \Theta_{\mathrm{s}}
$$

Solid phase shear viscosity

$$
\begin{aligned}
& \mu_{\mathrm{s}}=\mu_{\mathrm{s}, \text { kin }}+\mu_{\mathrm{s}, \mathrm{col}}+\mu_{\mathrm{s}, \mathrm{fr}} \\
& \mu_{\mathrm{s}, \mathrm{kin}}=\frac{5 \rho_{\mathrm{s}} d_{\mathrm{s}} \sqrt{\pi \Theta_{\mathrm{a}}}}{48 \varepsilon_{\mathrm{s}}(1+e) g_{0}}\left[1+\frac{4}{5} g_{0} \varepsilon_{\mathrm{s}}(1+e)\right]^{2} \\
& \mu_{\mathrm{s}, \mathrm{col}}=\frac{4}{5 \sqrt{\pi}} \varepsilon_{\mathrm{s}} \rho_{\mathrm{s}} g_{0} d_{\mathrm{s}}(1+e) \sqrt{\Theta_{\mathrm{s}}} \\
& \mu_{\mathrm{s}, \mathrm{fr}}=\frac{p_{\mathrm{s}} \sin \phi}{2 \sqrt{I_{2 \mathrm{D}}}}
\end{aligned}
$$

Solid phase bulk viscosity

$$
\lambda_{\mathrm{s}}=\frac{4}{3 \sqrt{\pi}} \varepsilon_{\mathrm{s}} \rho_{\mathrm{s}} g_{0} d_{\mathrm{s}}(1+e) \sqrt{\Theta_{\mathrm{s}}}
$$

Radial distribution function

$$
g_{0}=\left[1-\left(\frac{\varepsilon_{\mathrm{s}}}{\varepsilon_{\mathrm{s}, \max }}\right)^{1 / 3}\right]^{-1}
$$

\section{Supplement S3. The derivation of structure-based drag model for the bubbling}

\section{fluidized beds.}

For a bubbling fluidized bed, the gas flow is separated into the emulsion phase and bubble phase. In a unit volume of gas-particles flow, the drag force $\left(F_{\text {Den }}\right)$ of flowing gas on particles in the emulsion phase is:

$$
F_{\text {Den }}=n_{\mathrm{e}} F_{\text {De }}=\frac{\left(1-f_{\mathrm{b}}\right)\left(1-\varepsilon_{\mathrm{e}}\right)}{\frac{p}{6} d_{\mathrm{p}}^{3}} F_{\mathrm{De}}=\frac{3}{4} C_{\mathrm{De}} \frac{\rho_{\mathrm{g}}}{d_{\mathrm{p}}}\left(1-f_{\mathrm{b}}\right)\left(1-\varepsilon_{\mathrm{e}}\right) U_{\mathrm{se}}^{2}
$$


Meanwhile, the drag force $\left(F_{\mathrm{Dbn}}\right)$ of bubbles on particles in the emulsion phase is:

$$
F_{\mathrm{Dbn}}=n_{\mathrm{b}} F_{\mathrm{Db}}=\frac{f_{\mathrm{b}}}{\frac{\pi}{6} d_{\mathrm{b}}^{3}} F_{\mathrm{Db}}\left(1-\varepsilon_{\mathrm{e}}\right) \frac{\rho_{\mathrm{p}}}{\rho_{\mathrm{e}}}=\frac{3}{4} f_{\mathrm{b}}\left(1-\varepsilon_{\mathrm{e}}\right) C_{\mathrm{Db}} \frac{\rho_{\mathrm{p}}}{d_{\mathrm{b}}} U_{\mathrm{sb}}^{2}
$$

Therefore, the total drag force $\left(F_{\text {Dn }}\right)$ on particles in a unit volume of bed is:

$$
F_{\text {Dn }}=F_{\text {Den }}+F_{\text {Dbn }}=\frac{3}{4} C_{\text {De }} \frac{\rho_{\mathrm{g}}}{d_{\mathrm{p}}}\left(1-f_{\mathrm{b}}\right)\left(1-\varepsilon_{\mathrm{e}}\right) U_{\mathrm{se}}^{2}+\frac{3}{4} f_{\mathrm{b}}\left(1-\varepsilon_{\mathrm{e}}\right) C_{\mathrm{Db}} \frac{\rho_{\mathrm{p}}}{d_{\mathrm{b}}} U_{\mathrm{sb}}^{2}
$$

On the other hand, according to the definition of averaged drag coefficient, the total drag force $\left(F_{\mathrm{D}}\right)$ can be also calculated by:

$$
F_{\mathrm{D}}=\frac{\left(1-\varepsilon_{\mathrm{g}}\right)}{\frac{\pi}{6} d_{\mathrm{p}}^{3}} \bar{C}_{\mathrm{D}} \frac{1}{2} \rho_{\mathrm{g}} \frac{\pi}{4} d_{\mathrm{p}}^{2} U_{\mathrm{s}}^{2}=\frac{3}{4}\left(1-\varepsilon_{\mathrm{g}}\right) \bar{C}_{\mathrm{D}} \frac{\rho_{\mathrm{g}}}{d_{\mathrm{p}}} U_{\mathrm{s}}^{2}
$$

Therefore, the equation for averaged drag coefficient $\bar{C}_{\mathrm{D}, \mathrm{BFB}}$ can be obtained by comparing (S3-3) with (S3-4):

$$
\bar{C}_{\mathrm{D}, \mathrm{BFB}}=C_{\mathrm{De}}\left(1-f_{\mathrm{b}}\right) \frac{\left(1-\varepsilon_{\mathrm{e}}\right)}{\left(1-\varepsilon_{\mathrm{g}}\right)}\left(\frac{U_{\mathrm{se}}}{U_{\mathrm{s}}}\right)^{2}+C_{\mathrm{Db}} f_{\mathrm{b}} \frac{\left(1-\varepsilon_{\mathrm{e}}\right)}{\left(1-\varepsilon_{\mathrm{g}}\right)} \frac{\rho_{\mathrm{p}}}{\rho_{\mathrm{g}}} \frac{d_{\mathrm{p}}}{d_{\mathrm{b}}}\left(\frac{U_{\mathrm{sb}}}{U_{\mathrm{s}}}\right)^{2}
$$

\section{Supplement S4. The derivation of structure-based drag model for the fast fluidized beds.}

To model the hydrodynamic behaviors of fast fluidized beds, the complex heterogeneous flow structures can be divided into three simple homogeneous phases, including the cluster phase, the dispersed phase and the inter phase. The drag force $\left(F_{\text {Dcn }}\right)$ of flowing gas on particles in a unit volume of cluster phase is:

$$
\begin{aligned}
F_{\mathrm{Dcn}}=n_{\mathrm{c}} F_{\mathrm{Dc}} & =\frac{f_{\mathrm{c}}}{\frac{\pi}{6} d_{\mathrm{c}}^{3}}\left[\frac{\left(1-\varepsilon_{\mathrm{c}}\right) \frac{\pi}{6} d_{\mathrm{c}}^{3}}{\frac{\pi}{6} d_{\mathrm{p}}^{3}}-\frac{\left(1-\varepsilon_{\mathrm{c}}\right) \pi d_{\mathrm{c}}^{2}}{2 \frac{\pi}{4} d_{\mathrm{p}}^{2}}\right] C_{\mathrm{Dc}} \frac{1}{2} \rho_{\mathrm{g}} \frac{\pi}{4} d_{\mathrm{p}}^{2} U_{\mathrm{sc}}^{2} \\
& =\left(1-\frac{2 d_{\mathrm{p}}}{d_{c}}\right) \frac{f_{\mathrm{c}}\left(1-\varepsilon_{\mathrm{c}}\right)}{\frac{\pi}{6} d_{\mathrm{p}}^{3}} C_{\mathrm{Dc}} \frac{1}{2} \rho_{\mathrm{g}} \frac{\pi}{4} d_{\mathrm{p}}^{2} U_{\mathrm{sc}}^{2}
\end{aligned}
$$


The drag force $\left(F_{\mathrm{Ddn}}\right)$ of flowing gas on particles in a unit volume of dispersed phase is:

$$
F_{\mathrm{Ddn}}=n_{\mathrm{d}} F_{\mathrm{Dd}}=\frac{\left(1-f_{\mathrm{c}}\right)\left(1-\varepsilon_{\mathrm{d}}\right)}{\frac{\pi}{6} d_{\mathrm{p}}^{3}} C_{\mathrm{Dd}} \frac{1}{2} \rho_{\mathrm{g}} \frac{\pi}{4} d_{\mathrm{p}}^{2} U_{\mathrm{sd}}^{2}
$$

The drag force $\left(F_{\text {Din }}\right)$ of flowing gas on cluster in a unit volume of inter phase is:

$$
F_{\text {Din }}=n_{\mathrm{i}} F_{\mathrm{Di}}=\frac{f_{\mathrm{c}}}{\frac{\pi}{6} d_{\mathrm{c}}^{3}} C_{\mathrm{Di}} \frac{1}{2} \rho_{\mathrm{g}} \frac{\pi}{4} d_{\mathrm{c}}^{2} U_{\mathrm{si}}^{2}
$$

Therefore, the total drag force $\left(F_{\mathrm{Dn}}\right)$ of gas on particles in a unit volume of bed is:

$$
\begin{gathered}
F_{\text {Dn }}=F_{\text {Dcn }}+F_{\text {Ddn }}+F_{\text {Din }}=\left(1-\frac{2 d_{\mathrm{p}}}{d_{c}}\right) \frac{f_{\mathrm{c}}\left(1-\varepsilon_{\mathrm{c}}\right)}{\frac{\pi}{6} d_{\mathrm{p}}^{3}} C_{\mathrm{Dc}} \frac{1}{2} \rho_{\mathrm{g}} \frac{\pi}{4} d_{\mathrm{p}}^{2} U_{\mathrm{sc}}^{2} \\
+\frac{\left(1-f_{\mathrm{c}}\right)\left(1-\varepsilon_{\mathrm{d}}\right)}{\frac{\pi}{6} d_{\mathrm{p}}^{3}} C_{\mathrm{Dd}} \frac{1}{2} \rho_{\mathrm{g}} \frac{\pi}{4} d_{\mathrm{p}}^{2} U_{\mathrm{sd}}^{2}+\frac{f_{\mathrm{c}}}{\frac{\pi}{6} d_{\mathrm{c}}^{3}} C_{\mathrm{Di}} \frac{1}{2} \rho_{\mathrm{g}} \frac{\pi}{4} d_{\mathrm{c}}^{2} U_{\mathrm{si}}^{2}
\end{gathered}
$$

Therefore, the equation for averaged drag coefficient $\bar{C}_{\mathrm{D}, \mathrm{CFB}}$ can be obtained by comparing (S3-4) with (S4-4):

$$
\bar{C}_{\mathrm{D}, \mathrm{CFB}}=C_{\mathrm{Dc}} f_{\mathrm{c}} \frac{\left(1-\varepsilon_{\mathrm{c}}\right)}{\left(1-\varepsilon_{\mathrm{g}}\right)}\left(1-2 \frac{d_{\mathrm{p}}}{d_{\mathrm{c}}}\right)\left(\frac{U_{\mathrm{sc}}}{U_{\mathrm{s}}}\right)^{2}+C_{\mathrm{Dd}}\left(1-f_{\mathrm{c}}\right) \frac{\left(1-\varepsilon_{\mathrm{d}}\right)}{\left(1-\varepsilon_{\mathrm{g}}\right)}\left(\frac{U_{\mathrm{sd}}}{U_{\mathrm{s}}}\right)^{2}+C_{\mathrm{Di}} \frac{f_{\mathrm{c}}}{\left(1-\varepsilon_{\mathrm{g}}\right)} \frac{d_{\mathrm{p}}}{d_{\mathrm{c}}}\left(\frac{U_{\mathrm{si}}}{U_{\mathrm{s}}}\right)^{2}
$$

\section{Notation}

\section{Latin letters}

$F_{\mathrm{Db}}$ The drag force of single bubble on single particle in emulsion phase, N

$F_{\text {Dbn }}$ The drag force of bubbles on particles in a unit volume of emulsion phase, $\mathrm{N} / \mathrm{m}^{3}$

$F_{\text {Dc }}$ The drag force of flowing gas on single particle in cluster phase, $\mathrm{N}$

$F_{\text {Den }}$ The drag force of flowing gas on particles in a unit volume of cluster phase, $\mathrm{N} / \mathrm{m}^{3}$

$F_{\text {Dd }}$ The drag force of flowing gas on single particle in dispersed phase, N

$F_{\text {Ddn }}$ The drag force of flowing gas on particles in a unit volume of dispersed phase, 
$\mathrm{N} / \mathrm{m}^{3}$

$F_{\text {De }}$ The drag force of flowing gas on single particle in emulsion phase, $\mathrm{N}$

$F_{\text {Den }}$ The drag force of flowing gas on particles in a unit volume of emulsion phase, $\mathrm{N} / \mathrm{m}^{3}$

$F_{\text {Di }}$ The drag force of flowing gas on single particle in inter phase, $\mathrm{N}$

$F_{\text {Din }}$ The drag force of flowing gas on particles in a unit volume of inter phase, $\mathrm{N} / \mathrm{m}^{3}$

$F_{\text {Dn }}$ The total drag force on particles in a unit volume of bed, $\mathrm{N} / \mathrm{m}^{3}$ 